\title{
A comparison of antigen-based rapid test and RT-PCR test to diagnose COVID-19 and its infectivity
}

\author{
Cokorda Agung Wahyu Purnamasidhi', Richard Christian Suteja², I Komang Hotra Adiputra², \\ Giovanca Verentzia Purnama², Ni Kadek Mulyantari³ ${ }^{3}$ I Ketut Agus Somia ${ }^{4}$ \\ ${ }^{1}$ Internal Medicine Department of Udayana University / Udayana University Hospital, Indonesia \\ ${ }^{2}$ Medical Faculty of Udayana University, Indonesia \\ ${ }^{3}$ Clinical Pathology Department of Udayana University / Sanglah General Hospital, Indonesia \\ ${ }^{4}$ Tropical and Infectious Diseases Division of Internal Medicine Department of Udayana University / \\ Sanglah General Hospital, Indonesia
}

\begin{abstract}
Background. Medical screening and diagnostic cost and equipment availability has been a major obstacle to supposedto-be extensive tracing, and overall, to the end of COVID-19 pandemic. Even though RT-PCR is the gold diagnostic standard, it is costly, lengthy, and may be unavailable in remote areas. Therefore, antigen-based COVID-19 rapid tests may be a solution to quickly detect and screen communities suspected of contracting COVID-19.

Objective. This paper aims to observe how reliable antigen-based COVID-19 rapid tests are compared to RT-PCR testing. Material and methods. An observational cross-sectional study was performed on 101 samples to find the specificity, sensitivity, and accuracy of antigen-based rapid testing compared to RT-PCR testing performed on every individual. Then, a pattern between $\mathrm{CT}$ values and duration between onset of symptoms and testing to antigen-based rapid test result was observed to find a cut-off value such that the person may be deemed safe to exit isolation.

Outcomes. A cut-off CT value of above $30.04(p<0.01$ ) with a sensitivity of $66.7 \%$ and specificity of $77.8 \%$ (moderate accuracy) obtained from ROC analysis showed negative results on antigen-based rapid tests. The tests showed an overall accuracy of $67.3 \%$, where results between the two tests were consistent.

Conclusion. Therefore, an estimated CT value of 30 was moderately proved to be used as a criterion to end isolation and presume the person no longer sheds SARS-CoV-2.
\end{abstract}

Keywords: antigen rapid test, COVID-19, RT-PCR, specificity, sensitivity

\section{INTRODUCTION}

A mysterious pneumonia outbreak was found in Wuhan, China in late 2019 (1). The disease was later known to be caused by SARS-CoV-2, a subfamily of orthocoronaviridae genus beta-coronavirus with a unique characteristic of crown-like spikes (2). This disease is most likely an evolution from coronaviruses found in pangolins or bats.(1,3). With uncontained massive migrations, SARS-CoV-2 easily spreads to other people through droplets and fomite, causing cases to rise exponentially $(1,4)$.
The most common symptom found in COVID-19 patients were fever, headache, cough, fatigue, myalgia, nausea, vomiting, diarrhoea, sore throat, and chest tightness (5). Moreover, loss of smell and taste, scientifically named as anosmia and ageusia, are unique symptoms found by patients infected by SARS-CoV-2 (6).

Containing COVID-19 requires a fast and reliable testing method to diagnose and screen suspected patients (7). Although real time-polymerase chain reaction (RT-PCR) is dubbed to be a gold diagnostic standard, processing sample taken takes hours and 
requires equipment not always readily available at remote diagnostic centres $(8,9)$. Therefore, antigen-based COVID-19 rapid testing was chosen as an alternative test to diagnose COVID-19 because of its availability, less time-consuming process (15-30 minutes), economical price, and because it doesn't require any special training $(7,8,10)$. Relying solely on RT-PCR as a diagnostic method do provide highly accurate results, however tracing may be hard because the process is time-consuming, therefore patients may infect other people during the waiting time. It may also overburden those in the poorer part of the community, and it may not always be available in remote regions where logistics and medical supply isn't abundant.

The test detects proteins excreted through respiratory tract, therefore also detecting viral shedding and infectivity (7). However, despite its lower cost and easy use, antigen-based rapid testing may show false negatives results. Studies found that the specificity of antigen-based rapid testing reaches or almost reaches $100 \%$, but sensitivity wasn't that good, with a literature stating $30.2 \%$ (9). Furthermore, its sensitivity will decrease when the cycle threshold (CT) value found on RT-PCR testing is above 30.00 or the equivalent of $9.4 \times 10^{3}$ copies $/ \mathrm{ml}$ (9). CT value is a semi-quantitative assessment of viral RNA concentration, where lower CT value indicates higher viral RNA concentrations $(11,12)$. An increase of 3.3 units in the CT value indicates a 10fold less target RNA under optimum conditions $(11,12)$. As a result, CT values served as an indirect indicator of relative viral load in patients $(11,12)$. Literature concluded that antigen-based rapid testing is therefore very useful for reducing the number of RT-PCR tests performed if it shows a positive result, but a finding of negative result requires an RT-PCR test (9).

\section{OBJECTIVES}

This observational cross-sectional study was conducted to find the specificity and sensitivity of antigen-based rapid testing of COVID-19 in patients hospitalized at Udayana University Hospital compared to RT-PCR testing. This result was further analysed to find a correlation between CT values of RT-PCR testing and antigen-based rapid testing result.

\section{MATERIAL AND METHODS}

We compared every RT-PCR and antigen-based COVID-19 rapid testing result in every individual sample collected from medical records in Udayana University Hospital. Inclusion criteria is all patients tested both by antigen-based COVID-19 rapid testing and RT-PCR with an interval of less than 24 hours. Although there were more patients tested by both antigen-based rapid testing and RT-PCR, these data were excluded due to a prolonged time interval between the tests. To count the specificity and sensitivity, a cross tabulation further analysed with a receiving-operating characteristic (ROC) and area under the curve (AUC) analysis was performed to evaluate the optimal cut off score. All statistical analysis was carried out with IBM SPSS Statistics 25. Ethical consent was obtained from Udayana University Hospital, numbered 1526/UN14.2.2.VII.14/ LT/2020/RSUNUD.

\section{RESULTS}

We observed 101 patients tested both by antigen-based COVID-19 rapid test and RT-PCR test. Patients tested range from 14 to 81 years old with a mean of 46.35 ( $S D \pm 15.4$ ) years old. Female ( $n=54$, $47.4 \%)$ predominates male $(n=47,41.2 \%$ ) in our sample pool. All of the patients tested present a minimum of one symptom, with few showing multiple symptoms. This may be an interpretation of real-life situations, where only people with symptoms and history of contact with a confirmed positive case tested themselves for early screening. Table 1 presents epidemiological data of patients, while figure 1 presents symptoms experienced by patients tested at Udayana University Hospital.

TABLE 1. Epidemiology of tested patients

\begin{tabular}{|c|c|c|c|c|}
\hline & & $\begin{array}{c}N \\
(n=101)\end{array}$ & $\%$ & Mean ( $\pm S D)$ \\
\hline \multicolumn{5}{|l|}{ Sex } \\
\hline & Male & 47 & $41.2 \%$ & \\
\hline & Female & 54 & $47.4 \%$ & \\
\hline \multicolumn{4}{|c|}{ Age (years old) } & $46.35( \pm 15.4)$ \\
\hline & $<20$ & 2 & $2.0 \%$ & \\
\hline & $20-29$ & 18 & $17.8 \%$ & \\
\hline & $30-39$ & 14 & $13.9 \%$ & \\
\hline & $40-49$ & 20 & $19.8 \%$ & \\
\hline & $50-59$ & 27 & $26.7 \%$ & \\
\hline & $>60$ & 20 & $19.8 \%$ & \\
\hline \multicolumn{4}{|c|}{$\begin{array}{l}\text { Duration between symptoms onset and testing } \\
\text { (days) }\end{array}$} & $13.32( \pm 6.23)$ \\
\hline & $0-6$ & 11 & $10.9 \%$ & \\
\hline & $7-13$ & 46 & $45.5 \%$ & \\
\hline & $14-20$ & 30 & $29.7 \%$ & \\
\hline & $21-27$ & 12 & $11.9 \%$ & \\
\hline & $18-34$ & 2 & $2 \%$ & \\
\hline
\end{tabular}

Table 2 presents a cross tabulation of both positive and negative results between both antigen-based rapid testing and reverse transcription-polymerase chain reaction testing.

A total of 18 subjects showed true positive while 50 subjects showed true negative. However, 30 pa- 


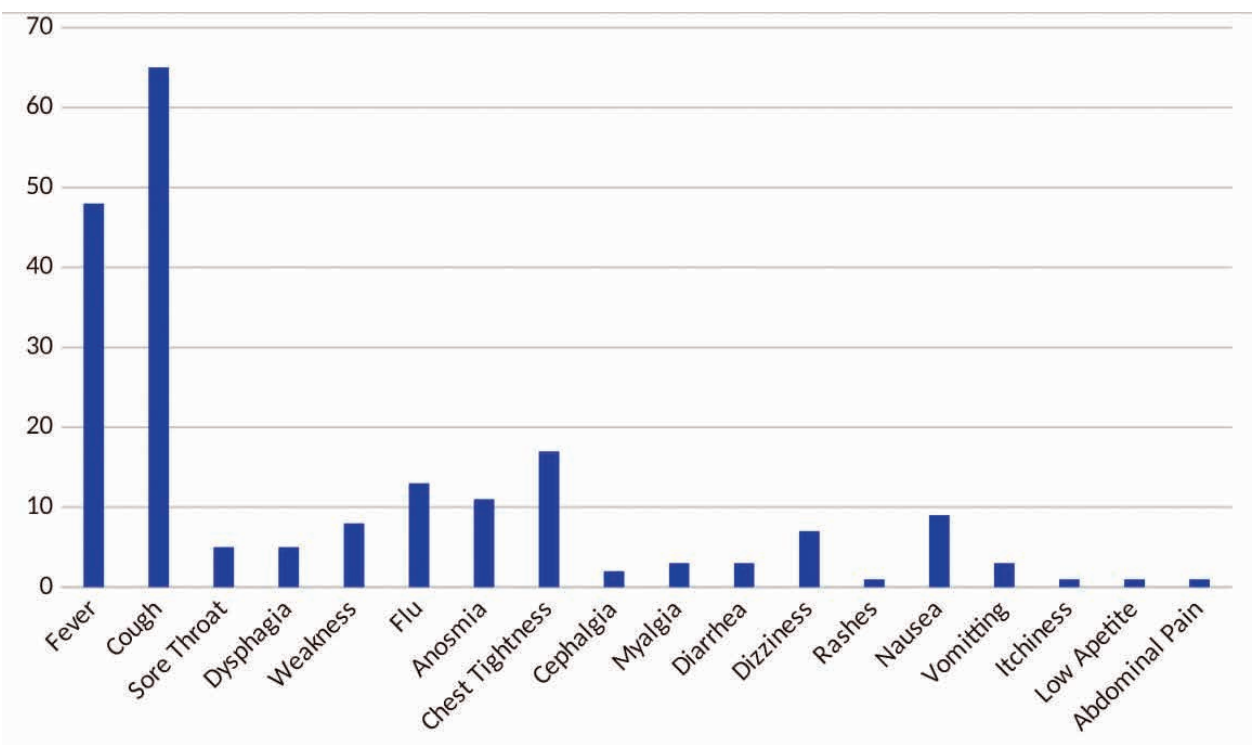

FIGURE 1. Symptoms shown by patients tested

TABLE 2. Cross tabulation between antigen-based rapid and RT-PCR

testing

\begin{tabular}{|c|c|c|c|c|}
\hline \multicolumn{2}{|c|}{} & \multicolumn{2}{|c|}{ Antigen-based rapid test } & \multirow{2}{*}{ Total } \\
\cline { 3 - 4 } \multicolumn{2}{|c|}{} & Positive & Negative & \\
\hline \multirow{3}{*}{ RT-PCR test } & Positive & $18(17.8 \%)$ & $30(29.7 \%)$ & \multirow{2}{*}{48 patients } \\
& & Male: 9 & Male: 8 & \\
& & Female: 9 & Female: 22 & \\
\cline { 3 - 4 } & Negative & $3(3 \%)$ & $50(49.5 \%)$ & 53 patients \\
& & Male: 3 & Male: 27 & \\
& & Female: 0 & Female: 23 & \\
\hline Total & & 21 patients & 80 patients & 101 patients \\
\hline
\end{tabular}

tients were shown to be positive during RT-PCR testing but were shown to be negative during antigen-based rapid testing. This number is significantly higher than its contra diagonal value, where only 3 patients were shown to be positive during antigen-based rapid testing but were shown to be negative during RT-PCR testing. From our findings, we found that antigen-based rapid testing had a sensitivity of $37.5 \%$, a specificity of $94.34 \%$, and an accuracy of $67.33 \%$. The fact that this antigen-based rap- id testing shows a low number of false positives indicates that this might be a reliable screening method for the mass.

We further draw the correlation between CT values shown by RT-PCR testing and the duration between onset of symptoms and testing. Figure 2 shows that the increase duration between onset of symptoms and testing is inversely proportional to CT values shown in RT-PCR positive test results.

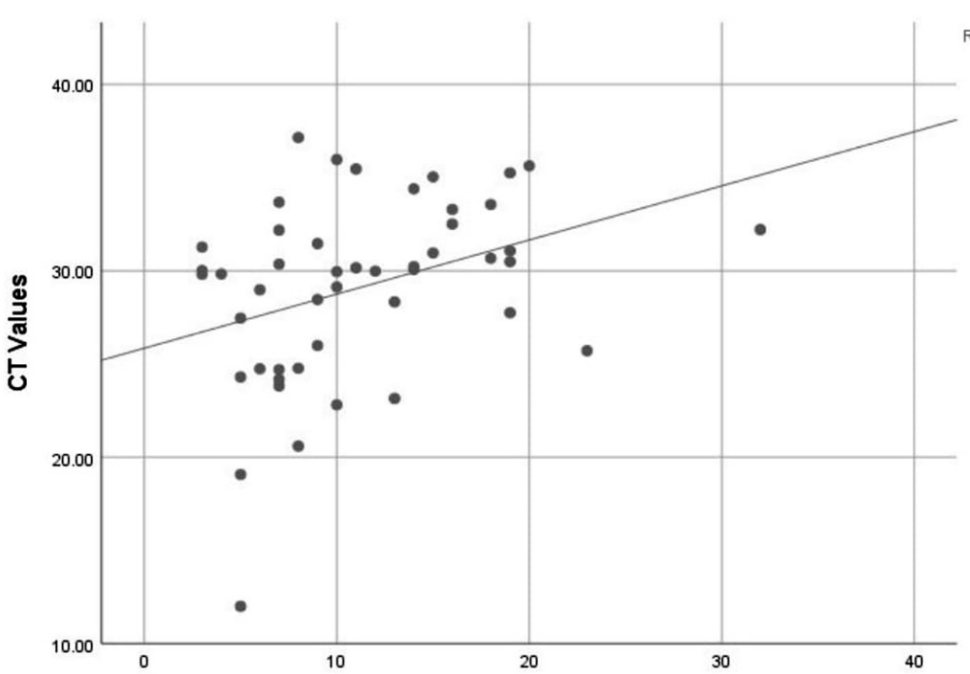

$R^{2}$ Linear $=0.129$

FIGURE 2. Scatter graph correlating between CT value and duration between onset of symptoms and testing 
We further analyse the correlation of antigen-based rapid testing and CT value to find a cutoff value in which the antigen-based rapid test starts to show negative results while RT-PCR test shows a positive result with high CT value. A linear regression found that the coefficient of determination is 0.129 and the line touches a CT value of 30.00 around 2 weeks post-onset of symptoms. This proves that by the end of 14 days of isolation post-onset of symptoms, antigen titres inside the patient's body decreased to a level where it may no longer shed due to shortage in numbers. We then correlate antigen-based COVID-19 rapid test's result, RT-PCR CT value, and duration between onset of symptoms and testing shown on table 3 . Though significant, we don't find any strong correlation between these three variables.

We performed a ROC analysis on CT values and to antigen-based rapid test result to find a cut-off value such that the person may be deemed safe to exit isolation. AUC found from ROC analysis correlating antigen-based rapid testing results and CT value of RT-PCR testing is $0.765(\mathrm{p}<0.01)$. It is shown on figure 3 that a cut-off CT value score of 30.04 with a $66.7 \%$ sensitivity and $77.8 \%$ specificity is where antigen-based rapid testing starts to show negative results. Therefore, a cut-off CT value score of above $30.04 \approx 30.00$ may be correlated to negative result of an antigen-based rapid test and is moderately accurate.

We then perform a second ROC analysis on duration between onset of symptoms and testing to antigen-based rapid test results to find a cut-off value such that the person may be deemed safe to exit isolation. AUC found from ROC analysis correlating antigen-based rapid testing results and CT value of RTPCR testing is $0.772(\mathrm{p}<0.01)$. It is shown on figure 4 that a cut-off duration of 9.5 days with a $73.3 \%$ sensitivity and $83.3 \%$ specificity is where antigen-based rapid testing starts to show negative results. There-

TABLE 3. Correlation between antigen-based COVID-19 rapid test result, RT-PCR CT value and duration between onset of symptoms and testing

\begin{tabular}{|l|l|r|r|r|}
\hline \multicolumn{2}{|l|}{} & $\begin{array}{c}\text { Antigen-based } \\
\text { COVID-19 } \\
\text { rapid testing }\end{array}$ & $\begin{array}{c}\text { Duration between } \\
\text { testing and onset of } \\
\text { symptoms }\end{array}$ & $\begin{array}{l}\text { RT-PCR CT } \\
\text { value }\end{array}$ \\
\hline \multirow{2}{*}{$\begin{array}{l}\text { Antigen-based COVID-19 } \\
\text { rapid testing }\end{array}$} & Pearson correlation & 1 & $.375^{* *}$ & $.439^{* *}$ \\
\cline { 2 - 5 } & Sig. (2-tailed) & & .009 & .002 \\
\hline $\begin{array}{l}\text { Duration between testing } \\
\text { and onset of symptoms }\end{array}$ & Pearson correlation & $.375^{* *}$ & 1 & $.343^{*}$ \\
\cline { 2 - 5 } & Sig. (2-tailed) & .009 & $.343^{*}$ & .017 \\
\hline RT-PCR CT value & Pearson correlation & $.439^{* *}$ & .017 & 1 \\
\cline { 2 - 5 } & Sig. (2-tailed) & .002 & & \\
\hline
\end{tabular}

*. Correlation is significant at the 0.05 level (2-tailed)

**. Correlation is significant at the 0.01 level (2-tailed)

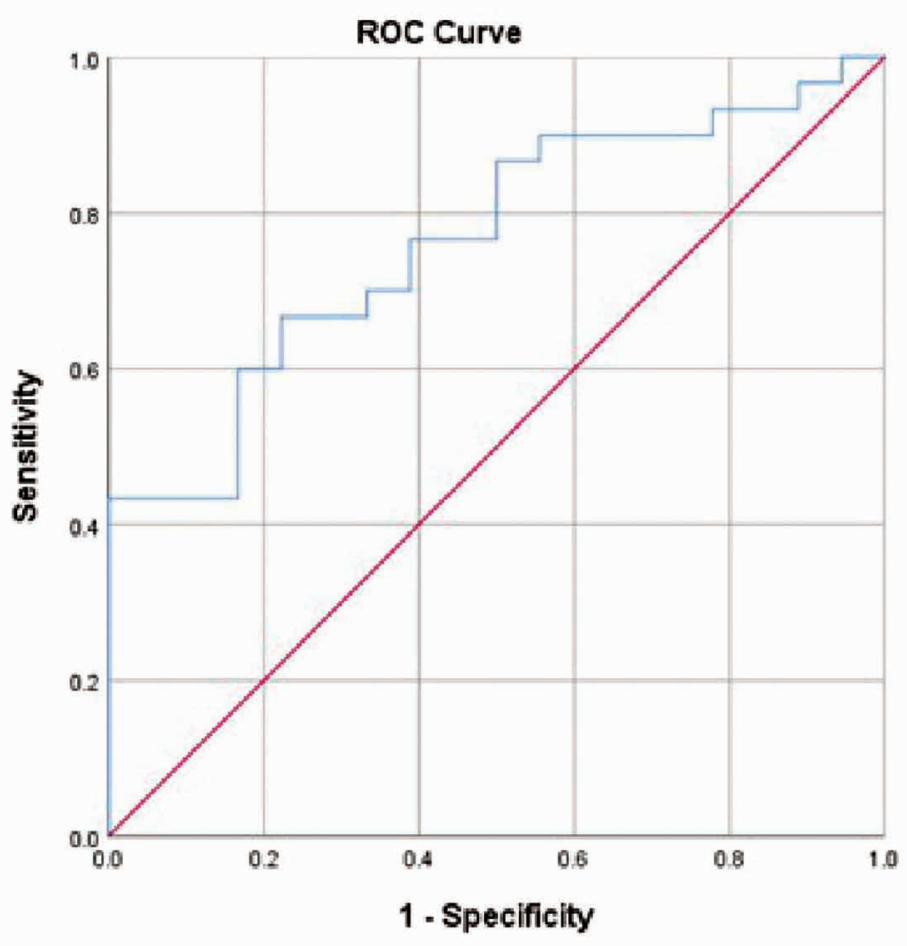

FIGURE 3. ROC curve and AUC correlating antigen-based rapid testing results and CT value of RT-PCR testing 


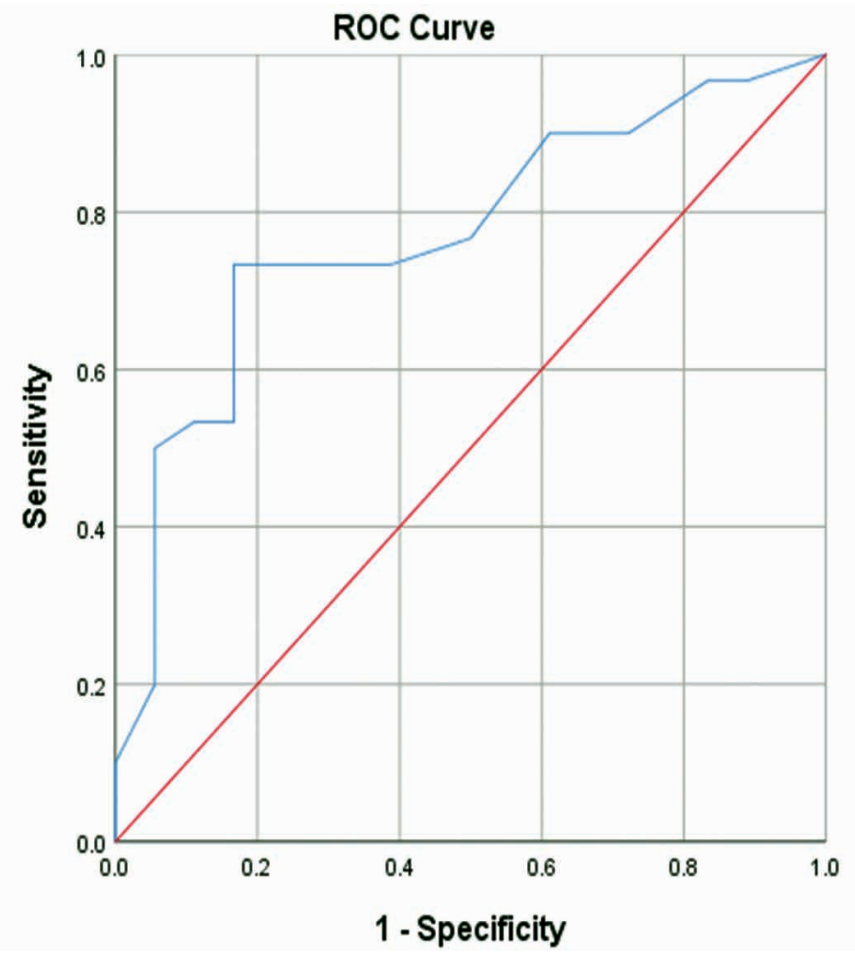

fore, a cut-off duration of above $9.5 \approx 10$ days may be correlated to negative result of an antigen-based rapid test and is moderately accurate. As antigen titres progressively drop to lower levels, directly proportional to the duration between onset of symptoms and testing, CT value of RT-PCR testing inversely increases.

Because studies stated that antigen-based rapid testing measures infectivity and viral shedding, therefore a negative antigen-based rapid test may be an indicator that the patient no longer sheds virus (13). This may further be translated that a CT value score of above $30.04 \approx 30.00$ or isolation of above $9.5 \approx 10$ days may be used as an moderate indicator that the patient no longer sheds virus and is safe to end isolation.

\section{DISCUSSION}

With the world in the brink of economic recession coupled by a swelling budget on mitigating healthcare impact of COVID-19 and economic incentives, the need for a reliable low-cost diagnostic test with high accuracy $(7,8,10)$. Without this diagnostic method, COVID-19 control may burden those in the poorer part of the community.

As RT-PCR test is a gold standard for diagnosing COVID-19, antigen-based rapid testing shifts more towards a substitute screening test rather than a substitute diagnostic test. In relation to existing studies, we found that antigen-based COVID-19 rapid test had a specificity of $94.34 \%$, lower than other literatures which found a specificity of $85.7-100 \%$ (14-19). The same is found with its sensitivity. Our
FIGURE 4. ROC curve and AUC correlating antigen-based rapid testing results and duration between onset of symptoms and testing

finding of $37.5 \%$ sensitivity is comparatively lower than other literatures, reporting a sensitivity of $50.6-93.3 \%$ (14-19). Our study found that antigen-based COVID-19 rapid test had an accuracy of $67.33 \%$, lower than other studies which found a comparatively higher result of $90.5 \%$ (19).

We compare our results with Merino et al. (19), which study scoped a total of 958 individuals with at least one symptom compatible with COVID-19 (n $=830$ ) or who had been in close contact with a confirmed COVID-19 case $(n=128)$. Merino et al. (19) found that antigen-based rapid test had a sensitivity of $90.5 \%$ (95\% CI 87.5-93.6) and a specificity of $98.8 \%$ (95\% CI 98-99.7). A significantly higher sensitivity is attributable to the lower duration interval between symptom onset or from exposure to testing. This causes a lower overall CT value, with a majority being below 25 .

Nash et al. (15) also recorded a sensitivity of $82 \%$ and specificity of $84.7 \%$, which again, is significantly higher than what we found in our study. Nash et al. (15) also supports the argument that repetitive rapid testing with actionable quarantine dramatically reduces disease spread, therefore supporting that antigen-based rapid testing may be used as a preliminary screening method. Porte et al. (16) recorded a sensitivity of $93.9 \%$ and specificity of $100 \%$.

Porte et al. (16) also recorded a high accuracy of $96.1 \%$. Again, this may be attributable to most individuals tested showing symptoms in less than 7 days after onset of symptoms (16). Most of patients tested also had some sort of symptoms (16) and CT value had a mean of 20 and IQR of 14.2-25.1 (16). However, it is also interesting to notice that a linear 
regression made by Porte et al. (16) showed a similar manner but displaced downwards compared to our regression. A lot of individuals tested in their study had a high viral load, shown by a large number of individuals with CT value below 15 .

Muhi et al. (17) recorded a sensitivity which is dependent upon the duration of symptoms reported, ranging from $77.3 \%$ in symptom onset less than 33 days to $100 \%$ in those less than 7 days. The specificity was $99.96 \%$, which is high and is aligned with other studies and our findings (14-19). Muhi et al. (17) also supported the use of antigen-based rapid testing as a tool for screening.

Bulilete et al. (18) included 1369 participants and found a sensitivity of $71.4 \%$ and specificity of $99.8 \%$. Bulilete et al. (18) also noted a high sensitivity in symptomatic patients, in patients tested 5 days since onset of symptoms, and in those with high viral load which translates to low CT-value.

\section{Limitations}

The authors were aware of the limits of this study. The data collected for this study is taken from a single health centre. Therefore, subjects and tools used might not be diverse enough to generalize the specificity and sensitivity of antigen-based rapid testing. Second, subjects tested were all symptomatic, therefore we cannot correlate results of antigen-based COVID-19 rapid testing and asymptomaticity. Third, there was no comparison with different brand or manufacturing site to obtain a more heterogenous data.

\section{CONCLUSIONS}

Antigen-based COVID-19 rapid testing is adequate as a screening method because of its low sensitivity (37.5\%) and high specificity (94.34\%). If an antigen-based COVID-19 rapid test shows a positive result, then a person may be clinically diagnosed suffering COVID-19. However, as negative results are prone to be false negatives, this may indicate the need for further testing, especially by RT-PCR as a gold standard.

Based on our analysis, an RT-PCR CT value of > 30.00 is a moderate predictor to antigen-based COVID-19 rapid test negativity, which means that no virus was shed and it is safe to end isolation.

It is to be noted that this may burden the nation economically furthermore, as suspects were required to take both tests. Therefore, a guideline towards a repetitive antigen-based COVID-19 rapid testing with intervals between may be a solution to lower the economic burden.

If repetitive antigen-based COVID-19 rapid testing are not possible - either due to economic or other factors - an isolation of more than 10 days or a CT we analysed two moderately accurate criteria may be used as a condition to end isolation:

\section{Acknowledgement}

The author would like to acknowledge Prof. DPG Purwa Samatra, Sp.S(K) as the director of Udayana University Hospital, Prof. I Ketut Suyasa, Sp.B, Sp. $\mathrm{OT}(\mathrm{K})$ as the dean of Medical Faculty of Udayana University and Dr. Herman Kosasih from INA-RESPOND.

Conflict of interest: none declared Financial support: none declared

\section{REFERENCES}

1. Suteja R, Novianti P, Dewi K, Widiyanti E, Wijayanti I, K C, et al. Migrant Workers of Indonesia: Transmission Channels of SARS-CoV-2 Amidst COVID-19 Pandemic in Indonesia. Int J Med Rev Case Reports. 2020;(0):1.

2. Wu YC, Chen CS, Chan YJ. The outbreak of COVID-19: An overview. J Chin Med Assoc. 2020 Mar;83(3):217-220.

3. Zhang $T$, Wu Q, Zhang Z. Probable Pangolin Origin of SARS-CoV-2 Associated with the COVID-19 Outbreak. Curr Biol. $2020 \mathrm{Apr}$ 6;30(7):1346-1351.e2.

4. Shereen MA, Khan S, Kazmi A, Bashir N, Siddique R. COVID-19 infection: Origin, transmission, and characteristics of human coronaviruses. Journal of Advanced Research. 2020;24:91-8.

5. Singhal T. A Review of Coronavirus Disease-2019 (COVID-19). Indian J Pediatr. 2020 Apr;87(4):281-286.

6. Mak PQ, Chung KS, Wong JSC, Shek CC, Kwan MYW. Anosmia and ageusia: Not an uncommon presentation of COVID-19 infection in children and adolescents. Pediatr Infect Dis J. 2020 Aug 1; 39(8):E199-200.

7. WHO. WHO provides one million antigen-detecting rapid diagnostic test kits to accelerate COVID-19 testing in Indonesia. 2021. Available at: https://www.who.int/indonesia/news/detail/17-03-2021-who-

provides-one-million-antigen-detecting-rapid-diagnostic-test-kits-toaccelerate-covid-19-testing-in-indonesia.

8. Mak GC, Cheng PK, Lau SS, Wong KK, Lau CS, Lam ET, et al. Evaluation of rapid antigen test for detection of SARS-CoV-2 virus. J Clin Virol. 2020 Aug 1;129:104500.

9. Scohy A, Anantharajah A, Bodéus M, Kabamba-Mukadi B, Verroken A, Rodriguez-Villalobos $\mathrm{H}$. Low performance of rapid antigen detection test as frontline testing for COVID-19 diagnosis. J Clin Virol. 2020 Aug 1;129:104455.

10. UNICEF Supply Division. Most affordable COVID-19 rapid diagnostic test now available. Available at: https://www.unicef.org/supply/ stories/most-affordable-covid-19-rapid-diagnostic-test-now-available.

11. Salvatore PP, Dawson P, Wadhwa A, Rabold EM, Buono S, Dietrich EA, et al. Epidemiological Correlates of Polymerase Chain Reaction Cycle Threshold Values in the Detection of Severe Acute Respiratory Syndrome Coronavirus 2 (SARS-CoV-2). Clin Infect Dis. 2021 Jun 1;72(11):E761-7.

12. Tom MR, Mina MJ. To Interpret the SARS-CoV-2 Test, Consider the Cycle Threshold Value. Clin Infect Dis. 2020 Nov 19;71(16):2252-4.

13. Vandenberg $O$, Martiny D, Rochas O, van Belkum A, Kozlakidis Z. Considerations for diagnostic COVID-19 tests. Nat Rev Microbiol. 2021 Mar 1;19(3):171-83. 
14. ICMR. Advisory on Use of Rapid Antigen Detection Test for COVID-19. 2020. Available at: https://www.icmr.gov.in/pdf/covid/strategy/ Advisory_for_rapid_antigen_test14062020.pdf.

15. Beatrice N, Anthony B, Ankita R, Miguel B, Nol S, Adam RG, et al. The impact of high frequency rapid viral antigen screening on COVID-19 spread and outcomes: a validation and modeling study. medRxiv. 2020 May 13.

16. Porte L, Legarraga P, Vollrath V, Aguilera X, Munita JM, Araos R, et al. Evaluation of a novel antigen-based rapid detection test for the diagnosis of SARS-CoV-2 in respiratory samples. Int J Infect Dis. 2020 Oct 1;99:328-33.

17. Muhi S, Tayler N, Hoang T, Ballard SA, Graham M, Rojek A, et al. Multi-site assessment of rapid, point-of-care antigen testing for the diagnosis of SARS-CoV-2 infection in a low-prevalence setting: A validation and implementation study. Lancet Reg Health West Pac. $2021 \mathrm{Apr} ; 9: 100115$.

18. Bulilete O, Lorente P, Leiva A, Carandell E, Oliver A, Rojo E, et al. Evaluation of the PanbioTM rapid antigen test for SARS-COV- 2 in primary health care centers and test sites. medRxiv. 2020 Nov.

19. Merino P, Guinea J, Muñoz-Gallego I, González-Donapetry P, Galán JC, Antona N, et al. Multicenter evaluation of the PanbioTM COVID-19 rapid antigen-detection test for the diagnosis of SARS-CoV-2 infection. Clin Microbiol Infect. 2021 Nov 20;27(5):758-61. 